\title{
Oncomir Microrna-346 is Upregulated in Ascending but Not Sigmoid Colon in Patients with Primary Sclerosing Cholangitis with Concurrent Ulcerative Colitis.
}

Agnieszka Kempinska-Podhorodecka ${ }^{1}$, Malgorzata Blatkiewicz ${ }^{1}$, Ewa Wunsch ${ }^{2}$, Lukasz $\mathrm{Krupa}^{3}, \mathrm{Krzysztof} \mathrm{Gutkowski}^{3}$, Piotr Milkiewicz ${ }^{2,4 *}$ and Malgorzata Milkiewicz ${ }^{1}$

${ }^{1}$ Department of Medical Biology, Pomeranian Medical University, Szczecin, Poland

${ }^{2}$ Translational Medicine Group, Pomeranian Medical University, Szczecin, Poland

${ }^{3}$ Department of Gastroenterology, Teaching Hospital No 1, Rzeszow, Poland

${ }^{4}$ Liver and Internal Medicine Unit, Medical University of Warsaw, Warsaw, Poland

*Corresponding author

Piotr Milkiewicz MD, MRCP(UK)

Liver and Internal Medicine Unit

Medical University of Warsaw

Banacha 1A

02-097 Warsaw, Poland

Tel.: (+48 22) 59925 45, 59925 46,

Fax: (+48 22) 5991545

e-mail: p.milkiewicz@wp.pl 


\begin{abstract}
Primary sclerosing cholangitis (PSC) is a cholestatic liver disorder frequently associated with ulcerative colitis (UC). Patients with PSC and UC have higher risk of colorectal neoplasia than patients with UC without PSC. Oncogenic properties of micro RNA 346 (miR-346) have been recently reported. In this study we investigated expressions of miR-346 and its two target genes i.e. the receptor of vitamin $\mathrm{D}(V D R)$ and the tumor necrosis factor $\alpha(T N F-\alpha)$, which are known to modulate carcinogenesis. Biopsies from ascending and sigmoid colon were obtained from patients with PSC with and without UC, patients with UC and healthy controls. MiR-346 expression was increased in ascending but not sigmoid colon of patients with PSC and UC when compared to other analyzed groups ( $p<0.001$ for all). In patients with UC an exceptionally low colonic expression of miRNA-346 was accompanied by the increase in VDR expression, and the extensive upregulation of $T N F-\alpha$ gene which protein product is known to be cytotoxic to tumor cells at high concentration. In summary, a substantial upregulation of miRNA-346 in ascending colon of patients with PSC and UC may be responsible for the inhibition of VDR and TNF- $\alpha$ signaling -pathway which may result in an inadequate suppression of neoplasia.
\end{abstract}

Key words: vitamin D receptor, cytokines, miR-346, primary sclerosing cholangitis, colorectal cancer

\title{
1. Introduction
}

Primary sclerosing cholangitis (PSC) is a chronic biliary disorder with a complex etiology characterized by a progressive destruction of biliary tract and consequently the liver through the mechanisms of autoimmunity and cholestasis. PSC mainly affects men and is commonly accompanied by inflammatory bowel diseases (IBD), predominantly ulcerative colitis (UC) [1]. Typically PSC exhibits an impaired hepatic excretion of bile acids (BA), which may be 
associated with elevated levels of fecal secondary BAs, mostly deoxycholic (DCA) and lithocolic acids (LCA) which were found to be positively associated colonic carcinomas [25]. Patients with PSC have an increased risk of developing primary bile duct cancer [6] and colorectal cancer (CRC) [7]. The risk of CRC development in PSC patients with concurrent IBD was found to be $14 \%$ at 10 years and $31 \%$ at 20 years, compared to a steady risk of $2.3 \%$ in patients without concurrent IBD [8], whereas in UC the overall prevalence of CRC patient is $3.7 \%$ [9]. Moreover, in the majority of IBD-PSC patients, who developed CRC, tumors are located in the right-sided colon in contrast to patients with sole IBD, what may suggest the differences in pathogenesis of CRC in these two groups of patients $[4,8,10,11]$.

Recently, the mechanism of CRC tumorigenesis has been linked with the area of microRNAs (miRNAs) [12]. MicroRNAs are a group of naturally occurring small non-coding RNA 18-25 nucleotides that are critical epigenomic regulators of gene expression and act either by translational repression or transcript degradation. Alterations in intracellular miRNAs were observed in numerous diseases including carcinoma. MiRNAs may possess eitheran tumorsuppressive or oncogenic activity depending on target genes [13-15]. Recently, miR-346 has been reported as oncogenic miRNAs (oncomiRs) in numerous cancers including prostate, lung breast and liver [16-21] but interestingly, not in colorectal neoplasia. Intriguingly, the level of miRNA-346 is consistently downregulated in the mucosal tissue of quiescent as well as active UC in comparison to healthy controls [22,23].

MicroRNA-346 inhibits, among other target genes, the expression of vitamin D receptor (VDR) via direct binding to a conserved target site within 3'UTR of VDR transcript [24]. VDR has two diverse biological roles, i.e. in calcium homesostasis and the autocrine-paracrine regulation of cell proliferation and differentiation. Antiproliferative effects of VDR have been demonstrated in a wide variety of cancer cell lines. Several lines of evidence suggest that VDR activation, which induces expression of cycle inhibitor p27(kip1), may be defensive against cancer [25,26] 
and low levels of vitamin $\mathrm{D}$ have been associated both with cancer and altered immune response[27-29]. Vitamin D down-regulates multiple signaling and metabolic pathways that are critical for T-cell activation and differentiation into pathogenic Th1 and Th17 subsets [30]. A reduction in epithelial VDR was suggested to affect the gut mucosal barrier and contributes to the development of IBD [31]. Moreover, the role of vitamin D in immune-mediated diseases appears to be closely associated with bacterial metabolism and chronic dysbiosis may trigger VDR dysfunction [25]. Tumor necrosis factor alpha (TNF- $\alpha)$ is a pro-inflammatory cytokine and key player in the pathogenesis of many inflammatory and autoimmune diseases. Recently, it was reported that miR-346 can indirectly modulate TNF- $\alpha$ expression either by inhibition of Bruton's tyrosine kinase (Btk) expression which is required for TNF- $\alpha$ production [32], or by inducing tristetraprolin, which destabilizes $T N F$ - $\alpha$ trascript [33].

Given that patients with PSC have an increased risk of the colorectal neoplasia in comparison to healthy subjects and UC patients, we investigated the expression of miR-346 and its two target genes including $V D R$ and $T N F$ - $\alpha$ in human colonic biopsies of patients with PSC or with UC.

\section{Results}

The substantial up-regulation of miR-346 expression was observed in the ascending colon of PSC-UC patients in comparison to all examined groups of patients $(p=0.0008 v s$. controls; $p=0001$ vs. PSC; $p=0.0001$ vs. UC; Figure 1A). In contrast, in PSC patients without concurrent UC the level of miRNA-346 was comparable to control values in the ascending colon (Figure 1A). On the contrary, in the sigmoid colon of PSC patients the expression of miRNA-346 was significantly increased ( $p=0.0001$ vs. controls; $p=0.0001 v s$. PSC-UC; $p=0.0001$ vs. UC; Figure 1B). Additionally, in patients with UC miRNA-346 expression was hardly detectable in both ascending and sigmoid colon ( $p=0.0001 v s$. controls, and $p=0.0001$ 
vs. controls, respectively; Figure 1A, B). A very low expression of miRNA-346 in both sigmoid and ascending colon of UC patients was accompanied by an extensive increase in the level of TNF- $\alpha$ mRNA (17-fold $v s$. controls, $p=0.001$; and 85-fold $v s$. controls, $p=0.01$, respectively, Figure 1E-F). This substantial enhancement in $T N-F \alpha$ expression was also evident in comparison to PSC and PSC-UC (Figure 1 E-F). And thus, the relative level of $T N F-\alpha$ in ascending colon of UC patients was 8.5-fold greater than that in PSC $(p=0.02)$ and 10-fold greater than that in PSC-UC ( $p=0.01$, Figure 1E). Similarly, in sigmoid colon $T N F$ $\alpha$ level was higher in UC than in PSC (17-fold increase, $p=0.002)$, or in PSC-UC (8-fold increase, $p=0.003$; Figure 1F).

In the ascending colon of PSC and PSC-UC patients there was a trend toward the increased expression of $T N F-\alpha$ mRNA (11-fold, $p=0.08$ vs. controls, and 10-fold, $p=0.09 v s$. controls, respectively, Figure 1E), but the level of this cytokine mRNA in the sigmoid colon was similar to control group values (Figure 1F).

In terms of VDR protein levels, both in ascending and sigmoid colon of PSC and PSCUC patients there was a high variability between patients and overall mean values were similar to controls (Figure 1C-D). On the other hand, in UC patients VDR protein expression was significantly increased in the sigmoid and ascending colon not only in comparison to controls (9.5-fold, $p=0.0009$, and 4.7-fold, $p=0.0003$, respectively), but also when compared to PSC (9fold, $p=0.001$ and 2.5-fold, $p=0.001$, respectively), and to PSC-UC (6-fold, $p=0.001$, and 2.5fold, $p=0.0008$, respectively; Figure 1C-D). 
ascending colon

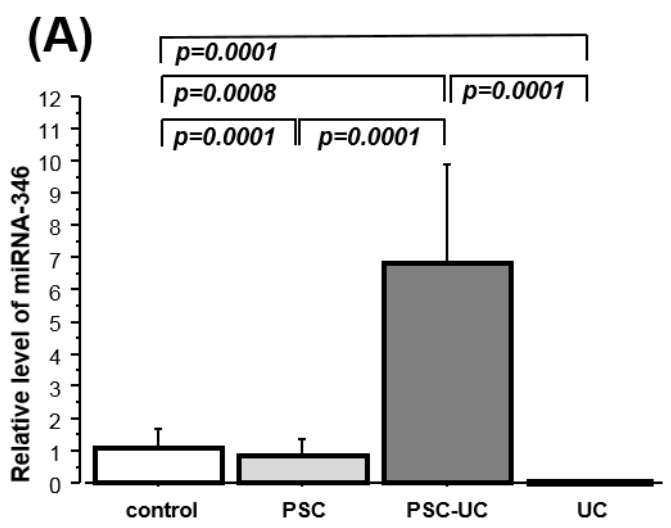

(C)
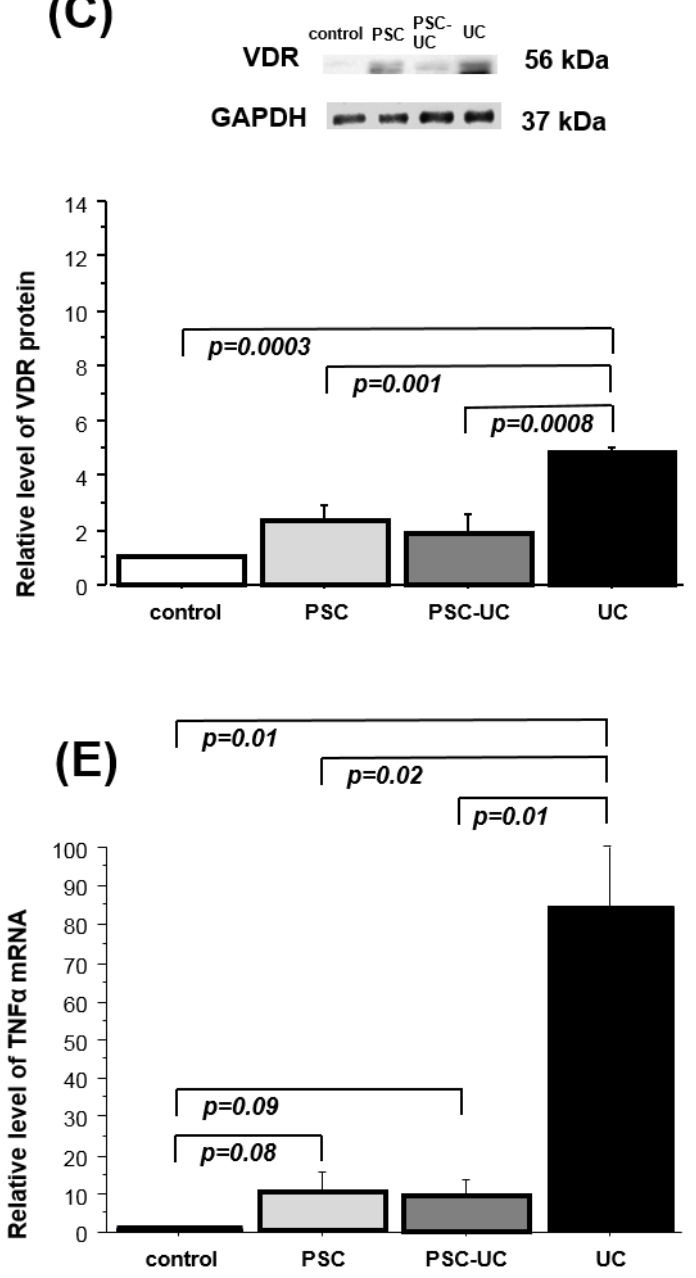

sigmoid colon

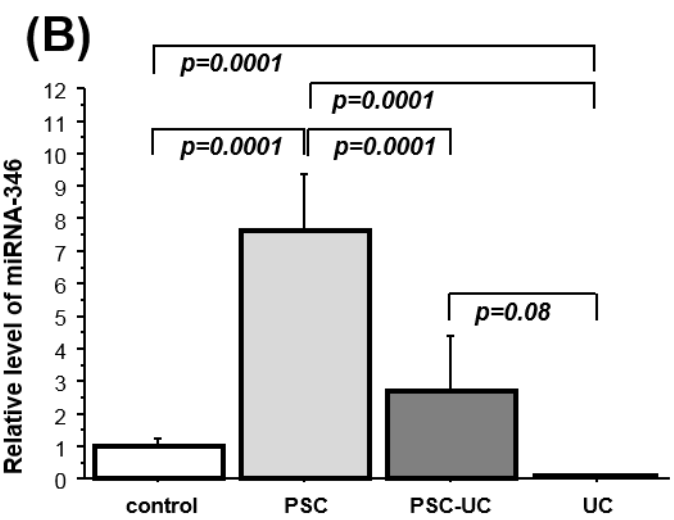

(D)
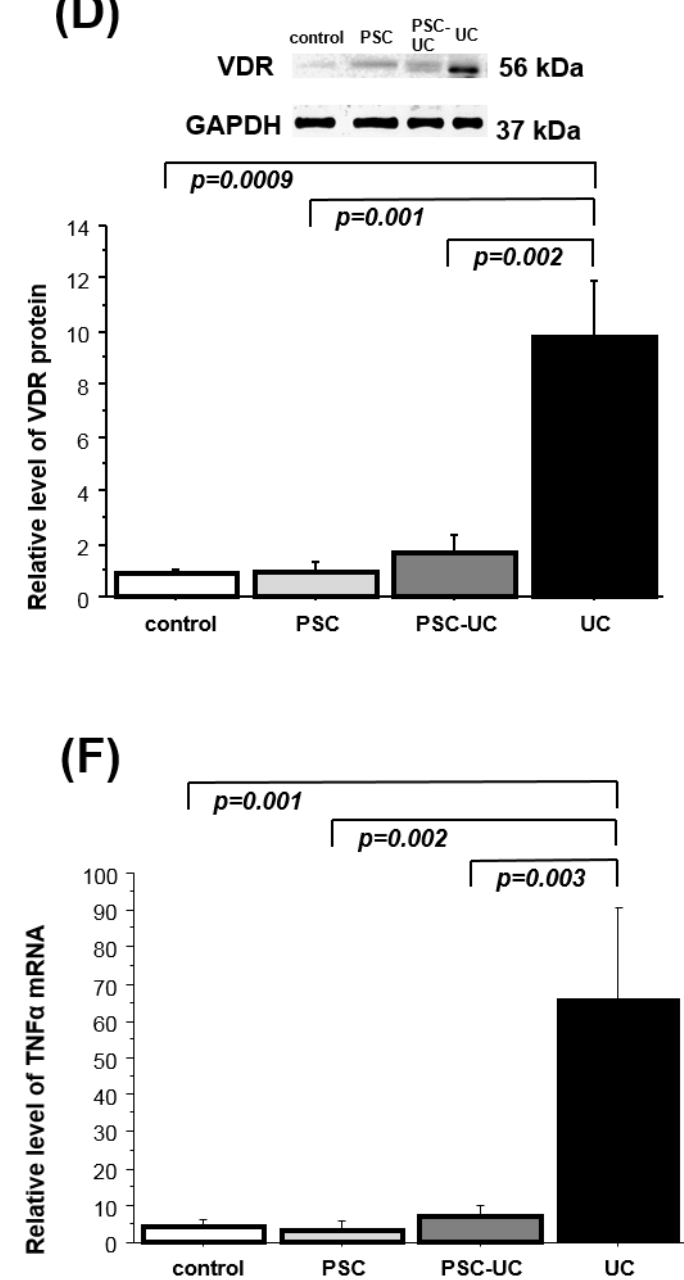

Figure 1. Expression of miRNA-346, VDR protein and TNF- $\alpha$ mRNA in ascending and sigmoid colon tissues. 
In the ascending colon (A, C, F) of patients with PSC-UC the miRNA-346 (A) expression was increased while the level of VDR protein $(\mathbf{C})$, and TNF- $\alpha$ mRNA (E) was not changed in comparison to controls. In the sigmoid colon $(\mathbf{B}, \mathbf{D}, \mathbf{F})$ expression of miRNA-346 (B) was significantly enhanced in PSC whereas VDR protein and TNF- $\alpha$ mRNA levels remained at control values. In both parts of colonic biopsies of patients with UC the miRNA-346 (A, B) expression was drastically suppressed, and it was accompanied by the enhanced expression of VDR protein $(\mathbf{C}, \mathbf{D})$, and mRNA TNF- $\alpha(\mathbf{E}, \mathbf{F})$. As reference microRNA served miRNA-191. Protein levels were determined by densitometry analysis after normalization to GAPDH as a control for loading. Levels of mRNA are presented as a n-fold change relative to controls after normalization to $18 \mathrm{~S}$ rRNA endogenous control. Results are representative of $\mathrm{n}=10$ independent experiments per group. Bars indicate the mean \pm SEM.

A significant negative correlation was observed between VDR protein and miRNA-346 in patients with UC in both parts of the colon (sigmoid colon: Rho=-0.5; and ascending colon: $R h o=-0.8)$. And VDR protein levels correlated positively with TNF- $\alpha$ mRNA in sigmoid colon of all the patients (PSC: $R h o=0.8$; PSC+UC: $R h o=0.9$; and UC: $R h o=0.7$ ) and in UC ascending colon $(R h o=0.9)$. Localization of VDR and TNF- $\alpha$ protein in control, PSC, and PSC-UC in human intestinal tissue is presented on Figure 2. 


\section{ascending colon}

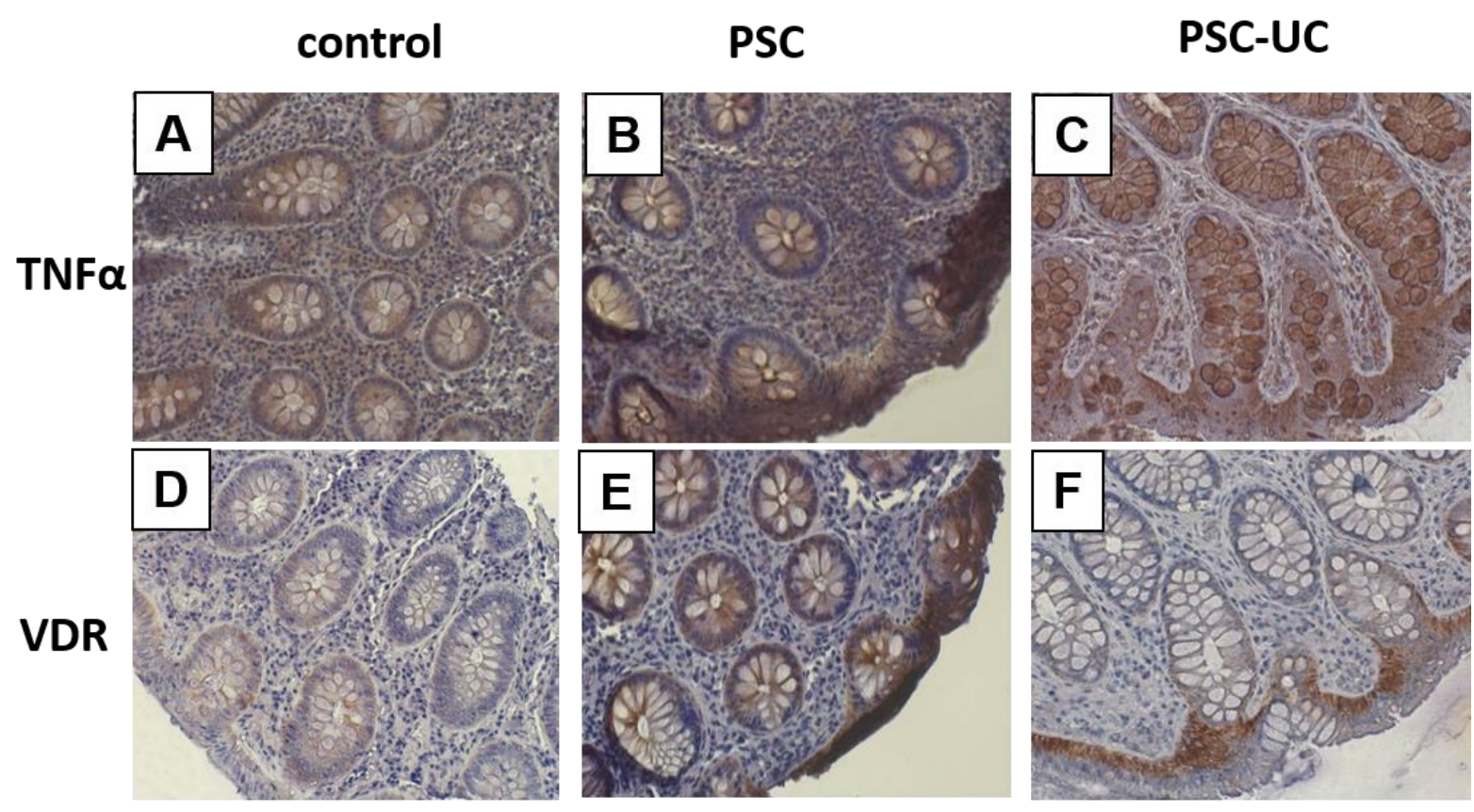

\section{sigmoid colon}
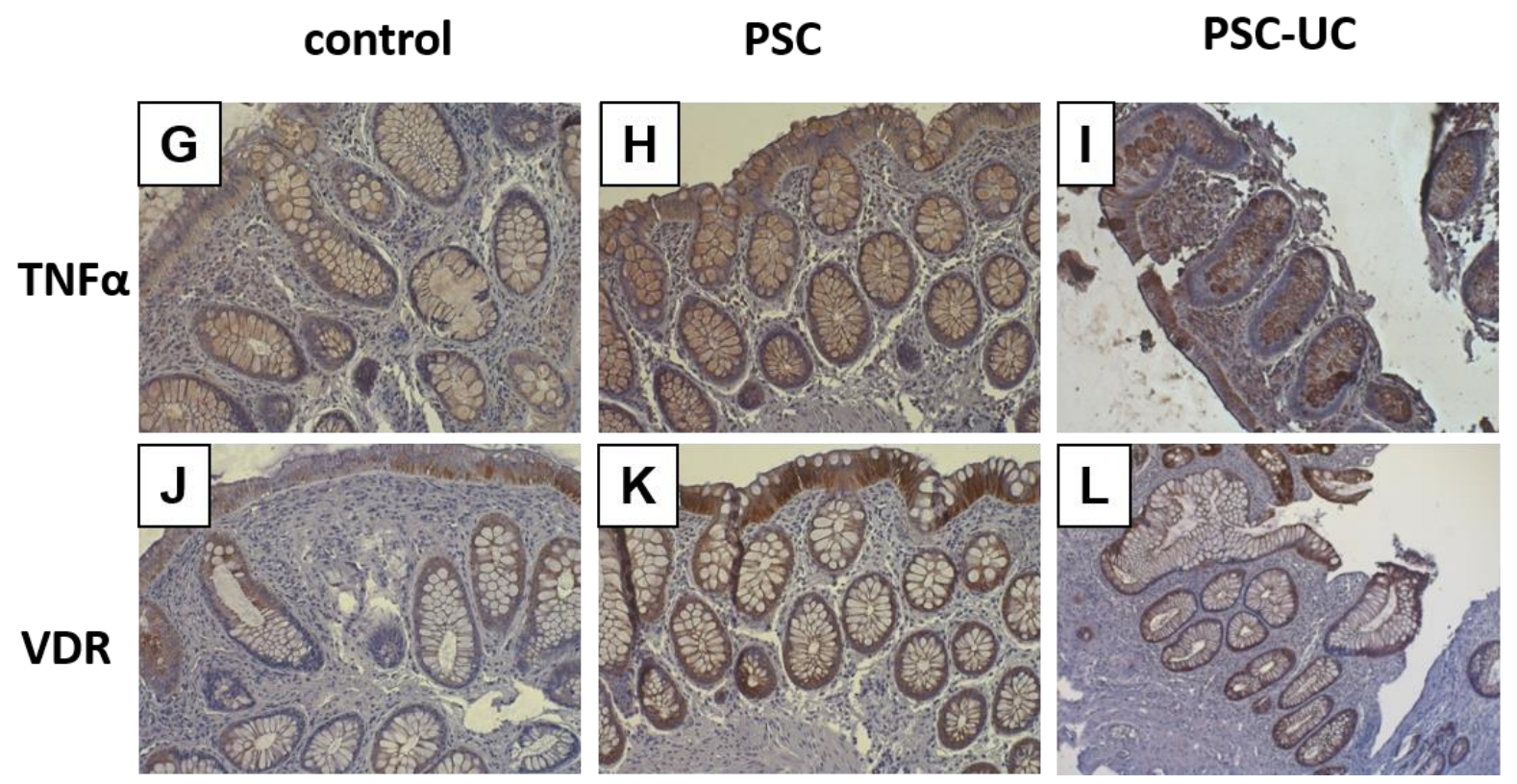

Figure 2. Immunohistochemical localization of VDR and TNF- $\alpha$ proteins in human intestinal tissue.

Representative immunostaining of colonic biopsies from normal control, PSC, and PSC-UC patients with anti-TNF- $\alpha$ antibodies $(\mathbf{A}, \mathbf{B}, \mathbf{C}$ and $\mathbf{G}, \mathbf{H}, \mathbf{I})$ and anti-VDR antibodies $(\mathbf{D}, \mathbf{E}, \mathbf{F}$ 
and $\mathbf{J}, \mathbf{K}, \mathbf{L})$. Brown staining indicate VDR or TNF- $\alpha$ protein in the epithelial cells. Nuclei were visualized by hematoxylin. Original magnification $\times 200$.

\section{Discussion}

The main finding of this study is a divergent expression of miR-346 in colonic tissues of patients PSC-UC in comparison to patients with UC alone. Another interesting observation is an inverse correlation between miR-346 and colonic TNF- $\alpha$ and VDR expressions.

Our study demonstrated the substantial increase in miR-346 expression in intestinal mucosa of PSC patients, but localizations of these changes were different depending on the part of the examined colon. Hence, in PSC-UC patients miR-346 was up-regulated in ascending colon while in PSC patient without concurrent UC the significant changes in miR-346 expression were observed in sigmoid colon. In PSC-UC patients increased levels of miRNA346 were seen in the part of colon where secondary BAs concentrations are the highest. It is known, that right proximal ascending colon is the predilection site for development of colonic malignancies in patients with PSC-UC and these patients tend to have more progressive tumors than patients with IBD without concomitant PSC [4]. Of note, miRNA-346 was hardly detected in both ascending and sigmoid colon from UC patients. These results are in agreement with previous studies, where miRNA-346 expression in colonic biopsies was reported to be consistently downregulated in both quiescent as well as active UC in comparison to healthy controls $[22,23]$.

Very low expression of miR-346 in both examined parts of colonic tissue of UC patients was associated with a very significant increase in the level of TNF- $\alpha$. These data are consistent with previous reports which showed independently either diminished expressions of miR-346 or increased levels of TNF- $\alpha$ in colons of patients with UC [22,23,34]. Some reports suggested, based on the animal and human data, that TNF- $\alpha$ promotes miR-346 expression [24]. However, 
in our study it was not the case, as we did not observe the induction of miR-346 in the presence of a very high level of TNF- $\alpha$ transcript. In contrast, in colonic tissues of PSC patients we demonstrated a negative association between miR-346 expression and $T N F-\alpha$ mRNA level. This observation can be supported by the report showing that TNF- $\alpha$ secretion in activated macrophages is controlled by miR-346 [33]. Our study revealed a distinct profile of TNF- $\alpha$ mRNA expression in PSC versus UC patients. In sigmoid colon of PSC patients both with or without concomitant UC, the level of $T N F-\alpha$ mRNA were comparable to control values in contrast to UC patients where the expression of $T N F-\alpha$ was drastically increased. In ascending colon, the level of $T N F-\alpha$ mRNA was increased in all PSC patients ( $p=0.08 v s$. control), however, it was too much lesser extent than in colonic mucosa of UC patients. TNF is a pleiotropic cytokine with dual roles in cancer biology as it has either pro- and anti-cancer activities [35]. This paradoxical roles depend on its local tissue concentration [36] hence TNF$\alpha$ is cytotoxic to tumor cells at high level, while at low level it fuels tumor-promoting inflammation and angiogenesis. The responses mediated by TNF- $\alpha$ are transduced via type 1 (TNFR-1) and type 2 receptors. TNFR-1 has a death membrane domain and mediates apoptosis, but besides this, it triggers the transcription of NF-kB and c-Jun, which are involved in cell proliferation and growth [37]. Association between chronic inflammation and the risk of colorectal cancer remains controversial. Several recent meta-analyses showed no evidence of the association between circulation level of TNF- $\alpha$ and the risk of colorectal cancer $[38,39]$. Moreover, anti-TNF- $\alpha$ therapies for the treatment of IBD, which aim to reduce level of this cytokine, have been suggested to have some undesirable effects such as induction of malignancy [40].

As the biological functions of miRNAs are known to be tissue specific and can be different under diverse pathophysiological conditions we examined the expression of another target of miR-346 i.e. VDR gene. Epithelial VDR signaling plays a critical role in maintaining 
the integrity of the mucosal epithelial barrier by suppressing inflammation-induced cells apoptosis [41-45]. The data on mucosal VDR expression in cholestatic patients are very limited. The VDR is known to be involved in the phenotypic features of PSC either by an indirect impact on intestinal permeability, or a direct modulation of innate immunity. In our previous study [46], we have demonstrated that the protein expressions of VDR decreased considerably in peripherial blood mononuclear cells and in livers of patients with PSC but its colonic expression was not investigated before. In this study we found that up-regulation of miR-346 in ascending colon of PSC-UC patients and in sigmoid colon of PSC patients was associated with reduction of VDR expression in comparison to UC patients. Additionally, we showed that very low expression of miRNA-346 in both parts of the colon of UC patients resulted in a substantial VDR induction. Down-regulation of VDR expression in a miR-346-dependent way was previously reported $[24,47]$. Our observations are in contradiction to a conclusion presented by Chen et al. [24,31] who hypothesized that inflammation characterized by an increased expression of pro-inflammatory cytokines (TNF- $\alpha$ and IL-1 $\beta$ ) down-regulates VDR expression, and they noted that TNF- $\alpha$ can suppress VDR by about $50-60 \%$ in active UC lesions [31]. In contrast, we observed the significant up-regulation of VDR protein level in colonic mucosa of UC patients which was accompanied by the substantial increase in TNF- $\alpha$ expression, but was negatively correlated with miR-346 expression (Figure 3).

In summary, the present study provides a novel insight into miRNA-346-depended modulation of TNF- $\alpha$ and VDR expression in the large intestine of PSC patients. The substantial upregulation of miRNA-346 in ascending colon of PSC-UC patients may be responsible for the inhibition of VDR and TNF- $\alpha$ signaling which may result in an inadequate suppression of neoplasia. Further investigation of the potential importance of colonic miRNA-346 in pathophysiological mechanisms of CRC development in patients with PSC are necessary to fully elucidate the role of this mRNA colonic carcinogenesis. 


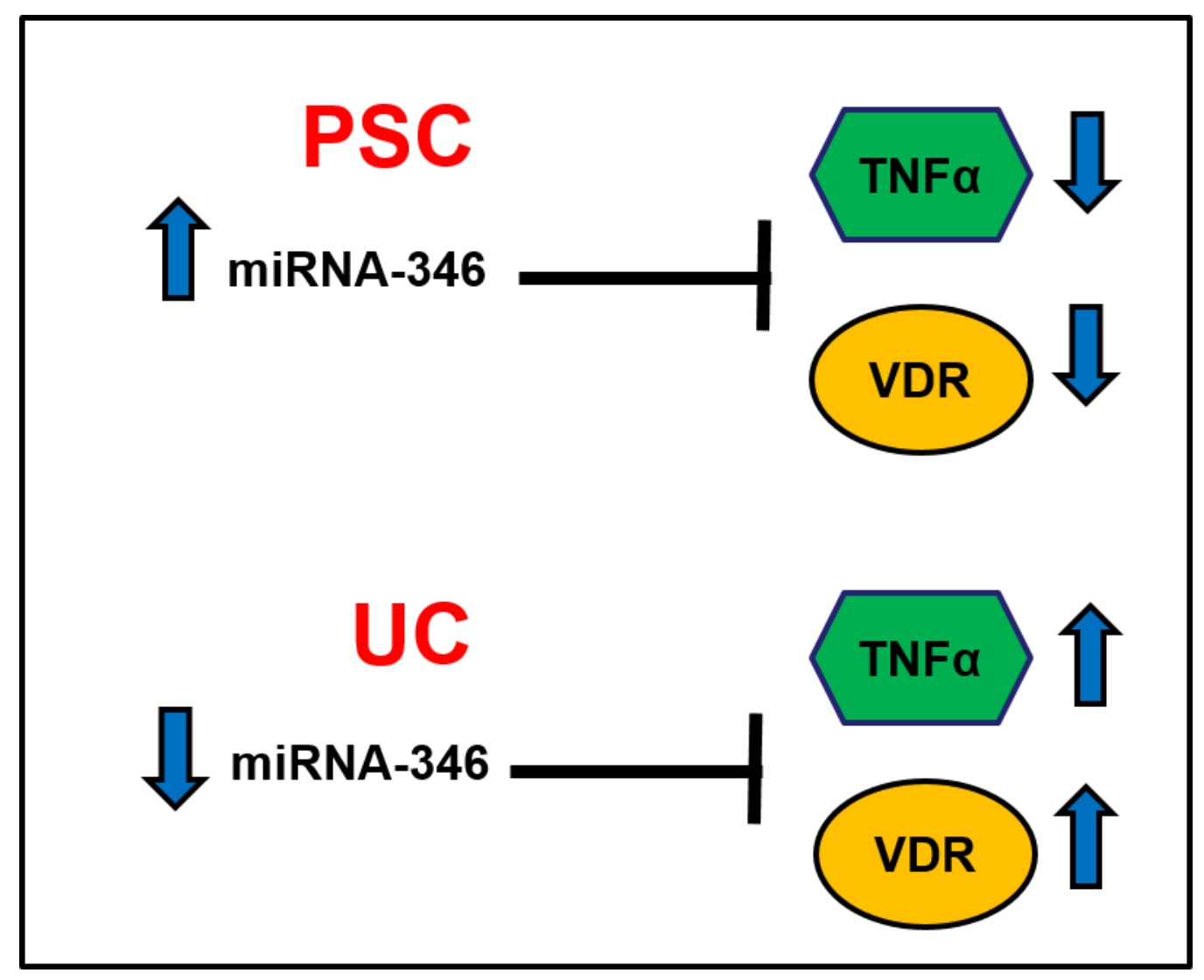

Figure 3. Schematic representation of iRNA-346-depended modulation of TNF- $\alpha$ and VDR expression in the large intestine.

\section{Patients and Methods}

\subsection{Patients Characteristics}

The study included 20 patients with PSC who underwent routine surveillance colonoscopies. Based on histopathological evaluation they were divided into two groups: the PSC group who had never been diagnosed with concomitant IBD ( $n=10,8$ males/ 2 females; mean age:30 \pm 9 years), and the PSC-UC group showing features of UC ( $n=10,7$ males/ 3 females; mean age:36 \pm 17 years). Additionally, 10 patients with UC (UC, 2 males and 8 female; mean age, $43.4 \pm 15$ years) were included in the study. The control group comprised 10 subjects ( 8 males and 6 females; mean age, $50 \pm 16$ years) who underwent colonoscopies for various indications and showed neither macroscopic nor microscopic abnormalities in their colons (Table 1). 
Clinical data were collected prior colonoscopy and tissue sampling. For molecular analysis, 3 biopsy specimens were obtained from ascending colon and from sigmoid colon. Each patient gave informed consent prior to participating in this study. The research protocol was approved by the Ethics Committee of Pomeranian Medical University and conformed to the ethical guidelines of the 1975 Declaration of Helsinki.

Table 1. Demographic and laboratory features of analyzed subjects

\begin{tabular}{|c|c|c|c|c|}
\hline Parameters & $\begin{array}{l}\text { Control } \\
(n=10)\end{array}$ & $\begin{array}{c}\text { PSC } \\
(n=10)\end{array}$ & $\begin{array}{c}\text { PSC + UC } \\
(n=10)\end{array}$ & $\begin{array}{c}\mathrm{UC} \\
(n=10)\end{array}$ \\
\hline Gender (M/F) & $6 / 4$ & $8 / 2$ & $7 / 3$ & $2 / 8$ \\
\hline Age $($ mean \pm SD) & $50 \pm 4$ & $30 \pm 9$ & $36 \pm 17$ & $43.4 \pm 15$ \\
\hline $\mathrm{Hb}(\mathrm{mmol} / \mathrm{l}$, mean $\pm \mathrm{SD})$ & - & $13.5 \pm 9.0$ & $13.1 \pm 1.8$ & - \\
\hline $\begin{array}{l}\text { Bilirubin }(\mu \mathrm{mol} / 1, \\
\text { mean } \pm \mathrm{SD})\end{array}$ & - & $10.1 \pm 3.6$ & $25.3 \pm 31.5$ & - \\
\hline $\operatorname{ALP}(\mathrm{U} / 1$, mean $\pm \mathrm{SD})$ & - & $159.4 \pm 108.7$ & $303.6 \pm 284.7$ & - \\
\hline $\operatorname{GGTP}(\mathrm{U} / 1$, mean $\pm \mathrm{SD})$ & - & $302.4 \pm 332.3$ & $381.8 \pm 187.0$ & - \\
\hline $\operatorname{ALT}(\mathrm{U} / 1$, mean $\pm \mathrm{SD})$ & - & $103.4 \pm 86.8$ & $56.8 \pm 67.2$ & - \\
\hline
\end{tabular}

Abbreviations: PSC- primary sclerosing cholangitis, UC - ulcerative colitis.

\subsection{Tissue specimen preparation}

In preparation for future analyses, intestine tissue biopsies were (i) stored in RNA later for subsequent evaluation of mRNA expression (AM7021; Applied Biosystems, Carlsbad, CA, USA), (ii) fixed in neutral-buffered formalin for histological assessment, or (iii) immediately frozen in liquid nitrogen for proteomic examination. 


\subsection{RNA and miRNA Expression Analysis}

Total RNA was isolated using RNeasy Mini kit (Qiagen, Hilden, Germany) and cDNA synthesis was carried out using Superscript II RT kit (Invitrogen, Carlbad, CA, USA) according to the protocol previously described [48]. Expressions of specific genes were measured by 7500 Fast Real-Time PCR System (Applied Biosystems, Foster City, CA, USA) using human TaqMan Gene Expression Assays for TNF- $\alpha$ (Hs00174128_m1), and 18SRNA (Hs99999901_s1). MiR-346 cDNA synthesis was carried out using TaqMan Advanced miRNA cDNA synthesis kit (Applied Biosystems, USA) according to the manufacturer's protocol and expression of miR-346 (478046_mir) and reference microRNA, miR-191 (477952_mir) were measured using TaqMan ${ }^{\circledR}$ Advanced miRNA assays and TaqMan ${ }^{\circledR}$ Fast Advanced Master Mix (Applied Biosystems, USA). The fluorescence data were analyzed with 7500 Software v2.0.2. (Applied Biosystems, USA) and relative amounts of transcripts were calculated using the $2-\Delta \Delta \mathrm{Ct}$ formula.

\subsection{Western blot analysis}

Proteins were extracted by homogenization of tissue samples in Radioimmunoprecipitation assay buffer (RIPA buffer) containing protease inhibitor cocktail and PhosSTOP (Roche Diagnostic, Mannheim, Germany). After electrophoresis in SDS polyacrylamide gels the proteins were blotted into polyvinylidene difluoride (PVDF) membranes under semi-dry transfer conditions (Thermo Scientific, Rockford, IL, USA), followed by an exposure to primary and secondary antibodies. The following antibodies were used: anti-VDR (sc-13133), anti-GAPDH (sc-25778) (Santa Cruz Biotechnology, Santa Cruz, CA, USA), and secondary

peroxidase conjugated anti-mouse antibody (Code: 115-035-146, Jackson ImmunoResearch Inc.). Bands were visualized by chemiluminescence detection (Chemiluminescent HRP 
Substrate, Millipore, Billerica, MA, USA) and quantified using MicroChemi 2.0 System and GelQuant software (Maale HaHamisha, Jerusalem, Israel).

\subsection{Immunohistochemistry}

After deparaffinization and antigen unmasking with citrate buffer (pH6.0 for 30 min in $98{ }^{\circ} \mathrm{C}$ ) the sections were fixated in cold acetone for 5 minutes $\left(-20^{\circ} \mathrm{C}\right)$. Then the endogenous peroxide activity was blocked by the incubation in $3 \%$ methanolic hydrogen peroxide (30 minutes) followed by blocking of unspecific binding by Normal Horse Serum (Vector Laboratories, Burlingame, CA, USA). After washing anti-VDR (sc-13133, Santa Cruz) and anti-TNF- $\alpha$ (sc52746, Santa Cruz) were used as primary antibodies and biotinylated anti-mouse/anti-rabbit IgG (BA-1400, Vector Laboratories) served as secondary antibody. Reactions were visualized using ABC Vectastain and DAB kits (Dako, Agilent Technologies, Denmark). Additionally, tissue structures were visualized by Mayer's Hematoxylin staining (DAKO). The negative controls, in which the primary antibodies were omitted, were included in the study and uniformly demonstrated no reaction. Images were acquired with ZEISS Axio Imager Z2 microscope equipped with Zen Pro 2011acquisition program.

\subsection{Statistics}

The two-tailed Student's $t$-test was used to compare two groups; multiple groups' comparisons were performed with Fisher's exact test or ANOVA with the StatView Program (SAS Institute Inc., Cary, NC, USA). Correlation analyses were performed using the Spearman Rank method. Results were considered statistically significant when $p$-values were $<0.05$. Data are displayed as mean and SEM if not indicated otherwise.

\section{Abbreviations}


- PSC - primary sclerosing cholangitis

- IBD - inflammatory bowel diseases

- UC - ulcerative colitis

- BA - bile acids

- LCA - lithocolic acid

- CRC- colorectal cancer

- VDR - vitamin D receptor

- TNF- $\alpha$-tumor necrosis factor alpha

- Btk - Bruton's tyrosine kinase

\section{Author Contributions}

Conceptualization and Writing - Original Draft Preparation, A.K.P. and M.M.; Methodology, A.K.P. and M.B.; Investigation, E.W. and L.K. and K.G.; Supervision, P.M.

\section{Conflicts of Interest}

The authors declare no conflict of interest.

\section{References}

1. Weismuller, T.J.; Trivedi, P.J.; Bergquist, A.; Imam, M.; Lenzen, H.; Ponsioen, C.Y.; Holm, K.; Gotthardt, D.; Farkkila, M.A.; Marschall, H.U., et al. Patient age, sex, and inflammatory bowel disease phenotype associate with course of primary sclerosing cholangitis. Gastroenterology 2017, 152, 1975-1984 e1978.

2. Torres, J.; Pineton de Chambrun, G.; Itzkowitz, S.; Sachar, D.B.; Colombel, J.F. Review article: Colorectal neoplasia in patients with primary sclerosing cholangitis and inflammatory bowel disease. Aliment Pharmacol Ther 2011, 34, 497-508.

3. Bernstein, H.; Bernstein, C.; Payne, C.M.; Dvorakova, K.; Garewal, H. Bile acids as carcinogens in human gastrointestinal cancers. Mutat Res 2005, 589, 47-65.

4. Claessen, M.M.; Lutgens, M.W.; van Buuren, H.R.; Oldenburg, B.; Stokkers, P.C.; van der Woude, C.J.; Hommes, D.W.; de Jong, D.J.; Dijkstra, G.; van Bodegraven, A.A., et al. More right- 
sided ibd-associated colorectal cancer in patients with primary sclerosing cholangitis. Inflamm Bowel Dis 2009, 15, 1331-1336.

5. Bayerdorffer, E.; Mannes, G.A.; Richter, W.O.; Ochsenkuhn, T.; Wiebecke, B.; Kopcke, W.; Paumgartner, G. Increased serum deoxycholic acid levels in men with colorectal adenomas. Gastroenterology 1993, 104, 145-151.

6. Danese, S.; Semeraro, S.; Papa, A.; Roberto, I.; Scaldaferri, F.; Fedeli, G.; Gasbarrini, G.; Gasbarrini, A. Extraintestinal manifestations in inflammatory bowel disease. World $J$ Gastroenterol 2005, 11, 7227-7236.

7. Harnois, D.M.; Gores, G.J.; Ludwig, J.; Steers, J.L.; LaRusso, N.F.; Wiesner, R.H. Are patients with cirrhotic stage primary sclerosing cholangitis at risk for the development of hepatocellular cancer? J Hepatol 1997, 27, 512-516.

8. Claessen, M.M.; Vleggaar, F.P.; Tytgat, K.M.; Siersema, P.D.; van Buuren, H.R. High lifetime risk of cancer in primary sclerosing cholangitis. $J$ Hepatol 2009, 50, 158-164.

9. Eaden, J.A.; Abrams, K.R.; Mayberry, J.F. The risk of colorectal cancer in ulcerative colitis: A meta-analysis. Gut 2001, 48, 526-535.

10. Shetty, K.; Rybicki, L.; Brzezinski, A.; Carey, W.D.; Lashner, B.A. The risk for cancer or dysplasia in ulcerative colitis patients with primary sclerosing cholangitis. Am J Gastroenterol 1999, 94, 1643-1649.

11. Marchesa, P.; Lashner, B.A.; Lavery, I.C.; Milsom, J.; Hull, T.L.; Strong, S.A.; Church, J.M.; Navarro, G.; Fazio, V.W. The risk of cancer and dysplasia among ulcerative colitis patients with primary sclerosing cholangitis. Am J Gastroenterol 1997, 92, 1285-1288.

12. Mohammadi, A.; Mansoori, B.; Baradaran, B. The role of micrornas in colorectal cancer. Biomed Pharmacother 2016, 84, 705-713.

13. Hsu, K.W.; Fang, W.L.; Huang, K.H.; Huang, T.T.; Lee, H.C.; Hsieh, R.H.; Chi, C.W.; Yeh, T.S. Notch1 pathway-mediated microrna-151-5p promotes gastric cancer progression. Oncotarget 2016, 7, 38036-38051.

14. Takahashi, R.U.; Miyazaki, H.; Takeshita, F.; Yamamoto, Y.; Minoura, K.; Ono, M.; Kodaira, M.; Tamura, K.; Mori, M.; Ochiya, T. Loss of microrna-27b contributes to breast cancer stem cell generation by activating enpp1. Nat Commun 2015, 6, 7318.

15. Gao, J.; Li, N.; Dong, Y.; Li, S.; Xu, L.; Li, X.; Li, Y.; Li, Z.; Ng, S.S.; Sung, J.J., et al. Mir-34a-5p suppresses colorectal cancer metastasis and predicts recurrence in patients with stage ii/iii colorectal cancer. Oncogene 2015, 34, 4142-4152.

16. Chen, B.; Pan, W.; Lin, X.; Hu, Z.; Jin, Y.; Chen, H.; Ma, G.; Qiu, Y.; Chang, L.; Hua, C., et al. Microrna-346 functions as an oncogene in cutaneous squamous cell carcinoma. Tumour Biol 2016, 37, 2765-2771.

17. Guo, J.; Lv, J.; Liu, M.; Tang, H. Mir-346 up-regulates argonaute 2 (ago2) protein expression to augment the activity of other micrornas (mirnas) and contributes to cervical cancer cell malignancy. J Biol Chem 2015, 290, 30342-30350.

18. Zhu, J.; Wang, S.; Zhang, W.; Qiu, J.; Shan, Y.; Yang, D.; Shen, B. Screening key micrornas for castration-resistant prostate cancer based on mirna/mrna functional synergistic network. Oncotarget 2015, 6, 43819-43830.

19. Yan, H.L.; Li, L.; Li, S.J.; Zhang, H.S.; Xu, W. Mir-346 promotes migration and invasion of nasopharyngeal carcinoma cells via targeting brms1. J Biochem Mol Toxicol 2016, 30, 602-607.

20. Sun, C.C.; Li, S.J.; Yuan, Z.P.; Li, D.J. Microrna-346 facilitates cell growth and metastasis, and suppresses cell apoptosis in human non-small cell lung cancer by regulation of xpc/erk/snail/ecadherin pathway. Aging (Albany NY) 2016, 8, 2509-2524.

21. Yang, F.; Luo, L.J.; Zhang, L.; Wang, D.D.; Yang, S.J.; Ding, L.; Li, J.; Chen, D.; Ma, R.; Wu, J.Z., et al. Mir-346 promotes the biological function of breast cancer cells by targeting srcin 1 and reduces chemosensitivity to docetaxel. Gene 2017, 600, 21-28.

22. Fisher, K.; Lin, J. Microrna in inflammatory bowel disease: Translational research and clinical implication. World J Gastroenterol 2015, 21, 12274-12282. 
23. Fasseu, M.; Treton, X.; Guichard, C.; Pedruzzi, E.; Cazals-Hatem, D.; Richard, C.; Aparicio, T.; Daniel, F.; Soule, J.C.; Moreau, R., et al. Identification of restricted subsets of mature microrna abnormally expressed in inactive colonic mucosa of patients with inflammatory bowel disease. PLoS One 2010, 5.

24. Chen, Y.; Du, J.; Zhang, Z.; Liu, T.; Shi, Y.; Ge, X.; Li, Y.C. Microrna-346 mediates tumor necrosis factor alpha-induced downregulation of gut epithelial vitamin $d$ receptor in inflammatory bowel diseases. Inflamm Bowel Dis 2014, 20, 1910-1918.

25. Del Pinto, R.; Ferri, C.; Cominelli, F. Vitamin d axis in inflammatory bowel diseases: Role, current uses and future perspectives. Int J Mol Sci 2017, 18.

26. Wang, X.; Gocek, E.; Liu, C.G.; Studzinski, G.P. Micrornas181 regulate the expression of p27kip1 in human myeloid leukemia cells induced to differentiate by 1,25-dihydroxyvitamin d3. Cell Cycle 2009, 8, 736-741.

27. Dankers, W.; Colin, E.M.; van Hamburg, J.P.; Lubberts, E. Vitamin d in autoimmunity: Molecular mechanisms and therapeutic potential. Front Immunol 2016, 7, 697.

28. Feldman, D.; Krishnan, A.V.; Swami, S.; Giovannucci, E.; Feldman, B.J. The role of vitamin d in reducing cancer risk and progression. Nat Rev Cancer 2014, 14, 342-357.

29. Haussler, M.R.; Whitfield, G.K.; Kaneko, I.; Haussler, C.A.; Hsieh, D.; Hsieh, J.C.; Jurutka, P.W. Molecular mechanisms of vitamin d action. Calcif Tissue Int 2013, 92, 77-98.

30. Zeitelhofer, M.; Adzemovic, M.Z.; Gomez-Cabrero, D.; Bergman, P.; Hochmeister, S.; N'Diaye, M.; Paulson, A.; Ruhrmann, S.; Almgren, M.; Tegner, J.N., et al. Functional genomics analysis of vitamin $d$ effects on cd4+ $t$ cells in vivo in experimental autoimmune encephalomyelitis. Proc Natl Acad Sci U S A 2017, 114, E1678-E1687.

31. Liu, W.; Chen, Y.; Golan, M.A.; Annunziata, M.L.; Du, J.; Dougherty, U.; Kong, J.; Musch, M.; Huang, Y.; Pekow, J., et al. Intestinal epithelial vitamin d receptor signaling inhibits experimental colitis. J Clin Invest 2013, 123, 3983-3996.

32. Frenzel, L.; Semaan, N.; Alsaleh, G.; Wachsmann, D.; Gottenberg, J.-E.; Sibilia, J. A new mode of tnf-[alpha] inhibition by microrna (99.26). The Journal of Immunology 2009, 182, 99.2699.26.

33. Semaan, N.; Frenzel, L.; Alsaleh, G.; Suffert, G.; Gottenberg, J.E.; Sibilia, J.; Pfeffer, S.; Wachsmann, D. Mir-346 controls release of tnf-alpha protein and stability of its mrna in rheumatoid arthritis via tristetraprolin stabilization. PLoS One 2011, 6, e19827.

34. Olsen, T.; Goll, R.; Cui, G.; Husebekk, A.; Vonen, B.; Birketvedt, G.S.; Florholmen, J. Tissue levels of tumor necrosis factor-alpha correlates with grade of inflammation in untreated ulcerative colitis. Scand J Gastroenterol 2007, 42, 1312-1320.

35. Tse, B.W.; Scott, K.F.; Russell, P.J. Paradoxical roles of tumour necrosis factor-alpha in prostate cancer biology. Prostate Cancer 2012, 2012, 128965.

36. Fajardo, L.F.; Kwan, H.H.; Kowalski, J.; Prionas, S.D.; Allison, A.C. Dual role of tumor necrosis factor-alpha in angiogenesis. Am J Pathol 1992, 140, 539-544.

37. Smyth, M.J.; Cretney, E.; Kershaw, M.H.; Hayakawa, Y. Cytokines in cancer immunity and immunotherapy. Immunol Rev 2004, 202, 275-293.

38. Izano, M.; Wei, E.K.; Tai, C.; Swede, H.; Gregorich, S.; Harris, T.B.; Klepin, H.; Satterfield, S.; Murphy, R.; Newman, A.B., et al. Chronic inflammation and risk of colorectal and other obesityrelated cancers: The health, aging and body composition study. Int J Cancer 2016, 138, 11181128.

39. Zhang, X.; Liu, S.; Zhou, Y. Circulating levels of c-reactive protein, interleukin- 6 and tumor necrosis factor-alpha and risk of colorectal adenomas: A meta-analysis. Oncotarget 2016, 7 , 64371-64379.

40. Melmed, G.Y.; Targan, S.R. Future biologic targets for ibd: Potentials and pitfalls. Nat Rev Gastroenterol Hepatol 2010, 7, 110-117.

41. Fasano, A.; Shea-Donohue, T. Mechanisms of disease: The role of intestinal barrier function in the pathogenesis of gastrointestinal autoimmune diseases. Nat Clin Pract Gastroenterol Hepatol 2005, 2, 416-422. 
42. Watson, A.J.; Chu, S.; Sieck, L.; Gerasimenko, O.; Bullen, T.; Campbell, F.; McKenna, M.; Rose, T.; Montrose, M.H. Epithelial barrier function in vivo is sustained despite gaps in epithelial layers. Gastroenterology 2005, 129, 902-912.

43. Gibson, P.R. Increased gut permeability in crohn's disease: Is tnf the link? Gut 2004, 53, 17241725.

44. Edelblum, K.L.; Yan, F.; Yamaoka, T.; Polk, D.B. Regulation of apoptosis during homeostasis and disease in the intestinal epithelium. Inflamm Bowel Dis 2006, 12, 413-424.

45. Li, Y.C.; Chen, Y.; Du, J. Critical roles of intestinal epithelial vitamin d receptor signaling in controlling gut mucosal inflammation. J Steroid Biochem Mol Biol 2015, 148, 179-183.

46. Kempinska-Podhorodecka, A.; Milkiewicz, M.; Wasik, U.; Ligocka, J.; Zawadzki, M.; Krawczyk, M.; Milkiewicz, P. Decreased expression of vitamin d receptor affects an immune response in primary biliary cholangitis via the vdr-mirna155-socs1 pathway. Int J Mol Sci 2017, 18.

47. Martinez-Moreno, J.M.; Herencia, C.; Montes de Oca, A.; Munoz-Castaneda, J.R.; RodriguezOrtiz, M.E.; Diaz-Tocados, J.M.; Peralbo-Santaella, E.; Camargo, A.; Canalejo, A.; Rodriguez, M., et al. Vitamin $\mathrm{d}$ modulates tissue factor and protease-activated receptor 2 expression in vascular smooth muscle cells. FASEB J 2016, 30, 1367-1376.

48. Kopycinska, J.; Kempinska-Podhorodecka, A.; Haas, T.; Elias, E.; DePinho, R.A.; Paik, J.; Milkiewicz, P.; Milkiewicz, M. Activation of foxo3a/bim axis in patients with primary biliary cirrhosis. Liver Int 2013, 33, 231-238. 